\title{
GCIP functions as a tumor suppressor in non-small cell lung cancer by suppressing Id1-mediated tumor promotion
}

\author{
Kuan-yu Chen ${ }^{1}$, Chao-chung Chen ${ }^{2}$, Yau-lin Tseng ${ }^{4}$, Yi-chien Chang ${ }^{4}$ and Ming- \\ Chung Chang ${ }^{1,3}$ \\ ${ }^{1}$ Institute of Biotechnology, National Cheng Kung University, Tainan, Taiwan \\ 2 Department of Biotechnology, College of Medicine and Nursing, Hung Kuang University, Taichung, Tainan \\ ${ }^{3}$ Department of Nutrition, College of Medicine and Nursing, Hung Kuang University, Taichung, Tainan \\ ${ }^{4}$ Department of Surgery, National Cheng Kung University Medical College and Hospital, Tainan, Taiwan \\ Correspondence to: Ming-chung Chang, email: mcchang@mail.ncku.edu.tw
}

Keywords: GCIP, NSCLC, Id l, tumor suppressor

Received: April 4, $2014 \quad$ Accepted: June 6, $2014 \quad$ Published: June 7, 2014

This is an open-access article distributed under the terms of the Creative Commons Attribution License, which permits unrestricted use, distribution, and reproduction in any medium, provided the original author and source are credited.

\section{ABSTRACT}

Grap2 and cyclin D1 interacting protein (GCIP) has been recognized as a putative tumor suppressor, but the molecular mechanisms underlying its anti-tumor properties remain undefined. Here, we report that GCIP is frequently downregulated in nonsmall cell lung cancer (NSCLC) tissues. Binding assays indicated that inhibitor of DNA binding/differentiation 1 (Id1) interacts with GCIP in the nucleus. Ectopic GCIP expression in the highly invasive NSCLC cell line, H1299, inhibited proliferation, colony formation, invasion and migration, and increased susceptibility to anticancer drugs. Conversely, silencing GCIP expression in the minimally invasive NSCLS cell line, A549, increased proliferation, colony formation, invasion, and migration in vitro, and increased survival and resistance to anticancer drugs. GCIP also suppresses tumorigenicity of NSCLC cells in vivo and GCIP suppresses NSCLC progression is mediated in part by interfering with Id1 signaling, which was confirmed in conditionally induced stable cell lines. In addition, GCIP downregulates the expression of Id1, and GCIP and Id 1 are inversely expressed in NSCLC cell lines and specimens. Taken together, these results suggest that GCIP is a potential tumor suppressor in NSCLC and that suppression of Id1-mediated oncogenic properties may be a key mechanism by which GCIP can potently suppress NSCLC tumor progression.

\section{INTRODUCTION}

Lung cancer is a leading cause of cancer death worldwide. More than $80 \%$ cases are classified as nonsmall cell lung cancer (NSCLC), which is characterized by its poor prognosis and resistance to antineoplastic drugs both in vitro and in vivo $[1,2]$. Patients with NSCLC have a 5 -year survival rate of less than $15 \%$ [3]. To improve the survival of patients with NSCLC, it is important to elucidate the signaling pathways regulating both NSCLC tumor promotion and suppression to identify novel prognostic markers and potential therapeutic targets $[4,5]$.

The inhibitor of DNA binding/differentiation (Id) proteins belong to the dominant-negative helix-loop-helix (dnHLH) family of proteins, which lack a basic domain for DNA binding [6]. Among four types of Id proteins (Id1, Id2, Id3, and Id4), Id1 has been extensively studied in various cancers and is linked to tumorigenesis, as aberrant elevation of Id 1 has been found in over 20 types of human cancer [7]. Moreover, high Id1 expression levels are correlated with aggressive and high-grade cancer, as well as poor clinical outcome in different tumor types [813]. In addition, among the identified genes that mediated breast cancer metastasis to the lungs, Id1 was identified as one of the most active [14]. Furthermore, Id1 is a novel prognostic factor in NSCLC patients [15] and is a common mediator of NSCLC progression and metastasis in both smokers and nonsmokers [16].

GCIP is a $40-\mathrm{kDa}$ HLH leucine zipper protein, which is also recognized as a dnHLH protein [17] and 
was originally identified to be a human Grap2 and cyclin D-interacting protein [18] and also was recognized as a human homologue of the MAID protein (HHM) [19] and a D-type cyclin-interacting protein 1 (DIP1) [20]. Although several proteins, such as nuclear p29, Rad associated with diabetes (Rad), ribosomal phosphoprotein (P0), and oligodendrocyte transcription factor 1 (Olig 1), interact with GCIP [21-25], its physiological function remains largely undefined. Previous studies indicated that GCIP is expressed mainly in terminally differentiated tissues and might play an important role in controlling cell differentiation and proliferation [26]. In addition, overexpression of GCIP in mouse liver suppressed diethylnitrosamine (DEN)-induced liver tumors in transgenic mice [27], and mice lacking GCIP (Maid) expression in the liver are prone to earlier development of hepatocellular carcinomas (HCCs) and hepatocellular adenomas (HCAs) [28]. In addition, expression of GCIP was reduced in several cancer tissues with tumor progression and metastasis, including breast, prostate and colon tumor tissues [22]. Furthermore, decreased expression of GCIP correlates with poor patient prognosis in breast cancer [29].

Although GCIP is considered a putative tumor suppressor in breast, colon and liver cancers, its role in NSCLC tumor progression remains unknown. In this report, we evaluated the expression of GCIP in NSCLC and explored its role in NSCLC progression. Our results revealed that GCIP expression was significantly downregulated in NSCLC tissues, and the antitumor activity of GCIP was mechanistically mediated by the ability of GCIP not only to interact with Id1 but also to suppress its transcriptional activity, and thereby, increasing the susceptibility to chemotherapeutic agents.

\section{RESULTS}

\section{GCIP expression is significantly downregulated in invasive NSCLC tissues}

To investigate the role of GCIP in NSCLC progression, we first assessed its expression in NSCLC gene expression data sets. The Oncomine database revealed that GCIP is significantly downregulated in 58 primary NSCLC tissues, compared with adjacent normal tissues (Fig. 1A, $P<0.0001$ ). We then investigated GCIP
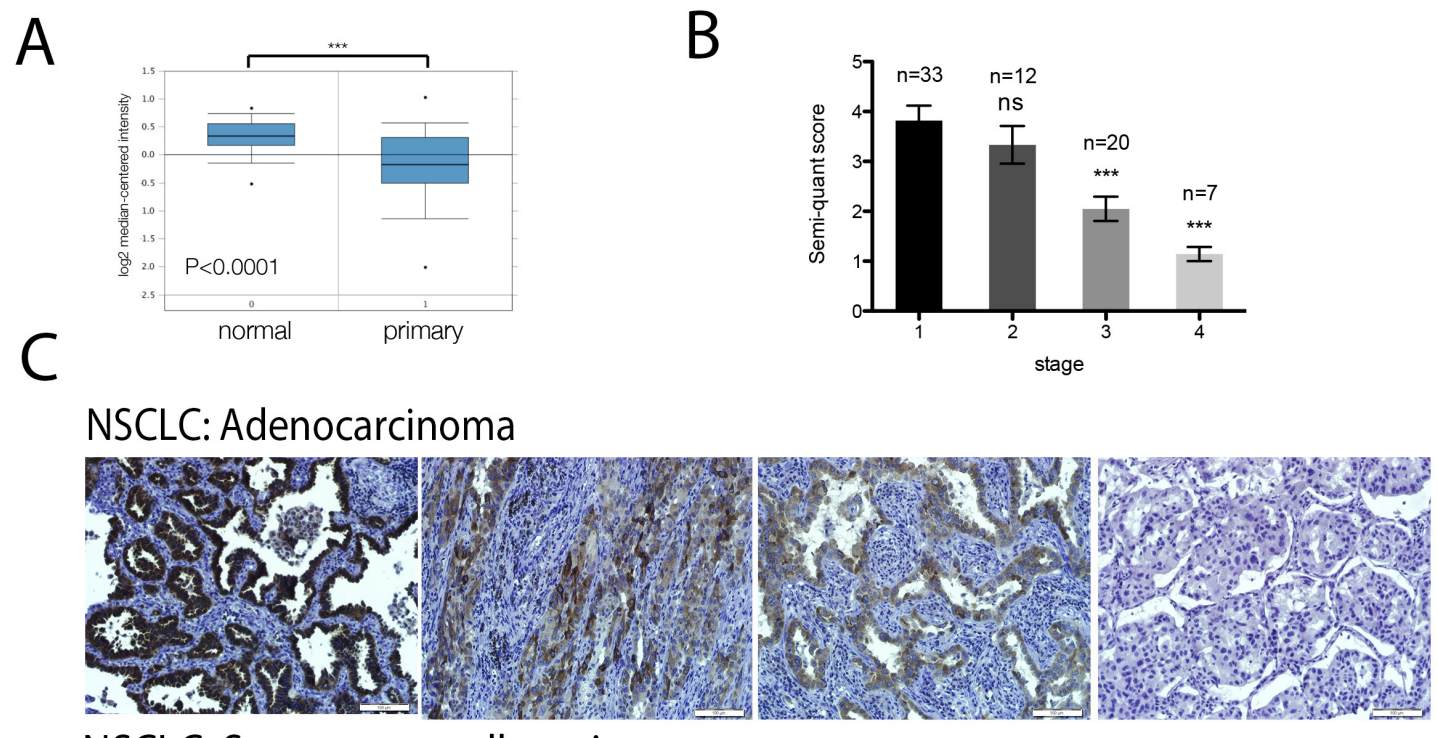

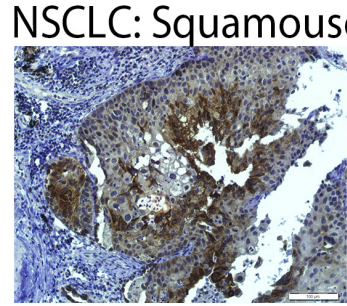

stage 1

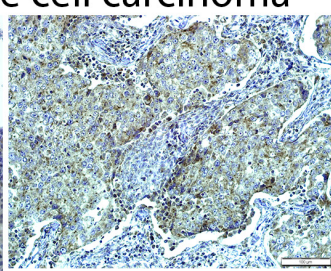

stage2

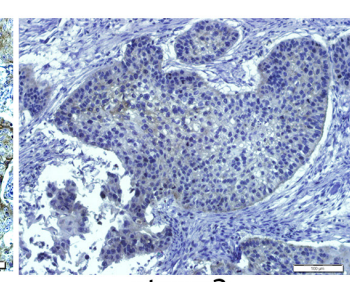

stage3

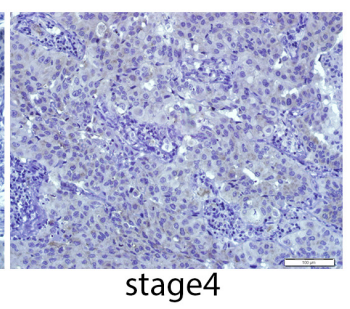

Figure 1: GCIP expression is significantly downregulated in lung cancer tissues. A, comparison of GCIP mRNA levels in normal lung and NSCLC tissues (data from the Oncomine database). ${ }^{* * *} P<0.0001$ based on Student's t-test analysis. B, GCIP expression in NSCLC lung tissue was 1.86-fold less in stage 3 tumors and 3.34-fold less in stage 4 tumors as compared to stage 1 NSCLC lung tumors. C, prognostic role of GCIP protein levels in NSCLC (adenocarcinoma and squamous cell carcinoma) specimens were examined by immunohistochemical staining (magnification x20). Scale bars, $100 \mu \mathrm{m}$. 
expression levels in 72 NSCLC specimens (comprised $68.1 \%$ adenocarcinoma and $31.9 \%$ squamous cell carcinoma) by Immunohistochemistry. The result revealed significant downregulation of GCIP in both the adenocarcinoma and the squamous cell carcinoma (Fig. 1B). In addition, GCIP expression levels were lower in stage IV tumors as compared to stage I tumor (Fig. 1C). These results suggest that GCIP plays a negative role in tumor progression and may be a valuable biomarker for NSCLC.

\section{Identification of Id1 as a GCIP-interacting protein}

To gain more insight into GCIP function, we isolated proteins that interact with it using full-length GCIP as the bait in a yeast two-hybrid system. Among the positive clones screened from a human cDNA library, two positive clones had high sequence similarity with Id1. To examine whether GCIP interacts with Id1 in mammalian cells, 293T cells were cotransfected with c-Myc-GCIP and Flag-Id1 expression vectors. As shown in Fig. 2A, FlagId1 was specifically coimmunoprecipitated using an antiMyc antibody followed by Immunoblotting with an anti-

A

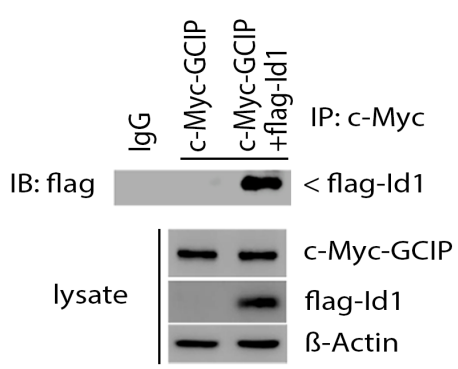

C

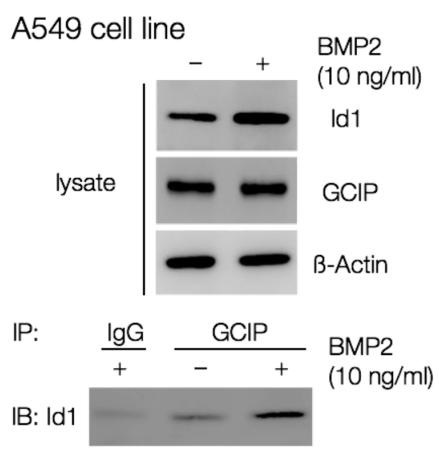

Flag antibody. Analysis of $293 \mathrm{~T}$ cell fractions revealed the c-Myc-GCIP Flag-Id1 complex was present mainly in the nucleus (Fig. 2B). In addition, immunofluorescence analysis indicated that GCIP and Id1 were co-localized in the nucleus of H1299 cells (Fig. S1). Furthermore, the interaction between the endogenous proteins was confirmed in A549 cells (Fig. 2C). Because Id1 expression could be induced by bone morphogenetic protein 2 (BMP2) [31], a markedly enhanced level of endogenous Id1 was coimmunoprecipitated in A549 cells stimulated with BMP2 for $24 \mathrm{~h}$ (Fig. 2C), further supporting the physical interaction between GCIP and Id1. To examine whether other Id proteins similarly interact with GCIP, four $\mathrm{pBIND}$ vectors containing full-length $\mathrm{Id} 1, \mathrm{Id} 2, \mathrm{Id} 3$, and Id4 cDNAs were constructed. Mammalian twohybrid assays indicated that Id1 exhibited the strongest interaction with GCIP followed by Id3; its interaction with Id 2 and Id4 was relatively weaker (Supplementary Fig. S2A). Similar results were obtained with GST pull-down assays (Fig. 2D). Using truncated protein constructions, mammalian two-hybrid assays revealed that GCIP requires its HLH domain to associate with Id1 (Supplementary Fig. S2B). Taken together, these results indicated that GCIP

B

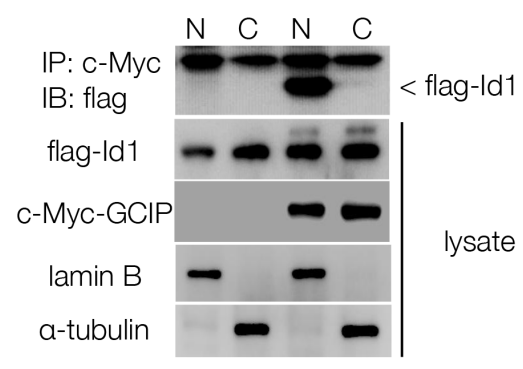

D

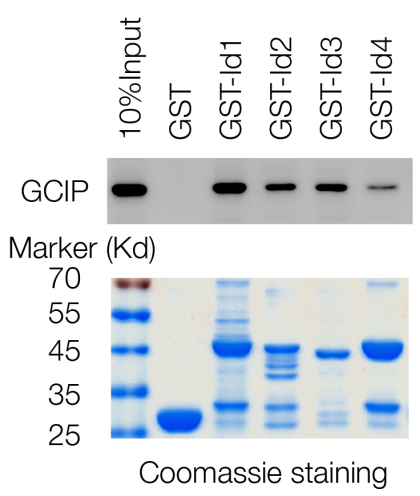

Figure 2: GCIP interacts with Id proteins in vitro and in vivo. A, co-immunoprecipitation assay with an anti-c-Myc antibody and immunoblotting using an anti-Flag antibody shows an interaction between Flag-Id1 and c-Myc-GCIP. B, nuclear localization of the c-Myc-GCIP/Flag-Id1 complex. 293T cells were transfected with vectors encoding GCIP and Id1. Cytoplasmic (C) and nuclear (N) fractions were prepared, and $100 \mu \mathrm{g}$ of proteins from each fraction were subjected to immunoprecipitation analysis with an anti-c-Myc antibody and immunoblotting using an anti-Flag antibody. C, BMP-2 treatment markedly induces endogenous Id1 expression in A549 cells. Immunoprecipitation assay using an anti-GCIP antibody shows increased interaction of GCIP and Id1 with BMP2 treatment. D, GSTpulldown assays were performed with in vitro-translated GCIP in the presence of GST-Id (Id1-4) fusion proteins. GST protein was used as a control. The immunoplexes were subjected to SDS-PAGE and probed with an anti-GCIP antibody. 
more specifically interacts with Id1, compared with the other Id proteins, and this interaction occurs in the nucleus. Furthermore, the HLH domain of GCIP is required for its association with Id1.

\section{GCIP inhibited and Id1 increased NSCLC proliferation in vitro}

In the less-invasive NSCLS cell lines, CL1-0 and A549. Western blot analysis indicated that relatively high levels of GCIP expression and relatively low levels of Id1expression were detected (Fig. 3A). In contrast, low levels of GCIP expression and high levels of Id1 expression were detected in the highly invasive NSCLC cell lines, CL1-5 and H1299 (Fig. 3A). To explore the functional significance of GCIP-Id1 interaction, two stable H1299 cells with ectopic expression of GCIP (H1299/GCIP clone 6, 9) and two A549 cells stably expressing GCIP shRNA (A549/shGCIP clone 3, 4) were next generated. Western blot analysis confirmed the high expression of GCIP in H1299/GCIP-9 and H1299/GCIP6 cells (Supplementary Fig. S3A) and reduced GCIP expression level in A549/shGCIP-3 cells as compared to controls (Supplementary Fig. S3B).

Consistent with a previous study [20], both H1299/GCIP-6 and -9 cells exhibited a decrease in cell proliferation (Fig. 3B, right). In contrast, both A549/ shGCIP-3 and -4 cells showed a significant increase in cell proliferation, when compared with respective controls (Fig. 3B, left). These results were further confirmed by colony formation assays (Fig. 3C). We also generated stable A549 cells that overexpress Id1 (A549/Id1) and stable H1299 cells that express shId1 (H1299/shId1) (Supplementary Fig. S3C and D). In accordance with a previous study by Yu-Jen Cheng et al. [32], A549/Id1 cells exhibited significantly increased cell proliferation, whereas H1299/shId1 cells displayed significantly decreased cell proliferation, when compared with respective controls (Supplementary Fig. 4A and B).

\section{GCIP suppresses NSCLC cell tumorigenicity in vitro and in vivo}

Boyden Chamber assays indicated that H1299/ GCIP-9 cells had markedly diminished migration and invasion, whereas A549/shGCIP-4 cells exhibited
A



C

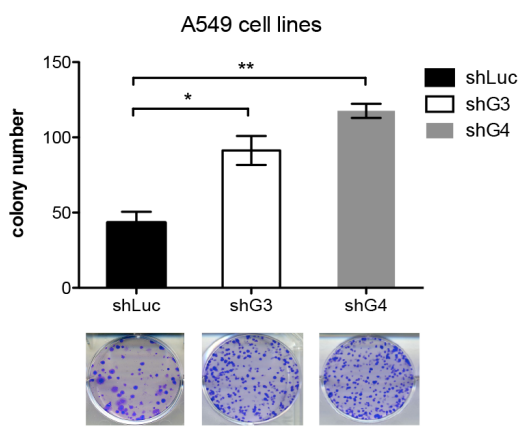

B
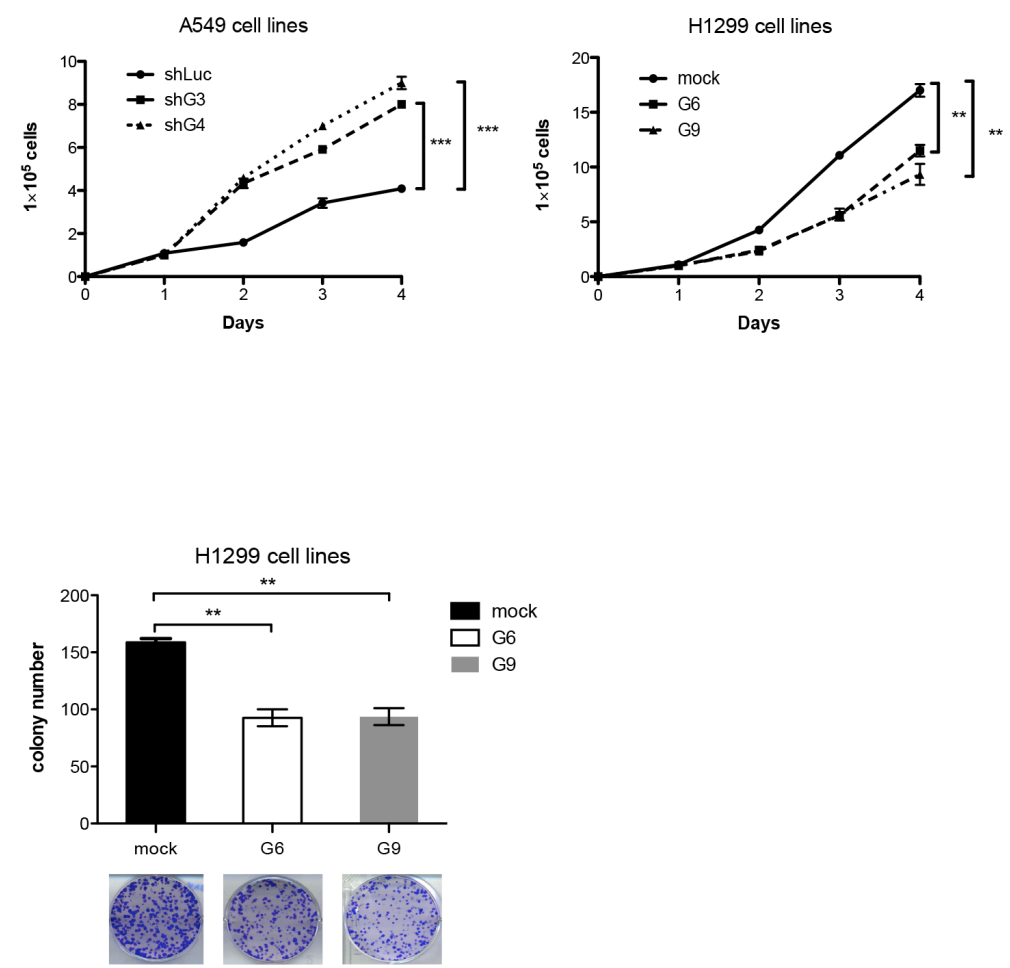

Figure 3: Effects of GCIP over or knockdown on NSCLC cell growth in vitro. A, endogenous expression of GCIP and Id1 in CL1-0, CL1-5, A549 and H1299, as measured by Western blot analysis. B, cell proliferation rate was determined by cell counting using the trypan blue exclusion assay. C, GCIP expression level-dependent decrease in colony formation. A549 (left) or H1299 (right) cell clones (500 cells/well) were seeded in a 6-well dish. After 2 weeks, the cells were stained with crystal violet and were counted by phase-contrast microscopy. All values are means $\pm \mathrm{SD}$ from at least three experiments. ${ }^{*} P<0.05 ;{ }^{* *} P<0.01 ;{ }^{* * *} P<0.001$ based on the Student's t-test. 
profoundly increased migration and invasion, when compared with respective controls (Fig. 4A and B). Whereas knockdown of Id1 diminished cell migration and invasion, its overexpression led to enhanced migration and invasion by H1299/shId1 cells and A549/Id1cells, respectively (Supplementary Fig. 5A and B). Consistent with the in vitro experiments, A549/shGCIP-4 cells had significantly greater tumor growth, whereas H1299/ GCIP-9 cells exhibited reduced tumor growth in NOC/ SCID mice (Fig. 4C). Specifically, the average tumor volume in mice bearing A549/shGCIP-4 cells was increased by 50\%, whereas mice bearing H1299/GCIP9 cells had tumor volumes that were decreased by $24 \%$, when compared to respective controls (Fig. 4C). Notably, immunohistochemical staining revealed that the H1299 GCIP-expressing tumors had significantly decreased Ki67staining relative to control tumors (Fig. 4D). Collectively, our data indicate that GCIP expression suppresses tumorigenicity of NSCLC cells in vitro and in vivo.

\section{GCIP suppresses NSCLC progression by interfering with Id1 signaling}

Previous studies demonstrated that Id1 expression was associated with activation of the Akt-related pathway and correlated with elevated expression of mesenchymal markers in NSCLC cells [16, 32]. Consistent with these studies, we observed a considerable reduction of phosphorylated-PI3K (Tyr467), phosphorylated-Akt (Ser473), NFkB/p65, the EMT inducers or markers (e.g., snail, slug, vimentin and fibronectin), and MMP9 expression levels in H1299/shId1 cells; a significant increase in p-PI3K (Tyr467), p-Akt (Ser473), NFkB/ p65, snail, slug, vimentin, fibronectin, and MMP9 expression levels in the A549/Id1 cells was also observed (Supplementary Fig. S6A and B). Furthermore, whereas both H1299/GCIP-6 and -9 cells had significantly decreased p-PI3K (Tyr467), p-Akt (Ser473), NFкB/p65, snail, slug, vimentin, fibronectin, and MMP9 expression levels, both A549/shGCIP-3 and -4 cells displayed significantly increased p-PI3K (Tyr467), p-Akt (Ser473), $\mathrm{NF \kappa B} / \mathrm{p} 65$, snail, slug, vimentin, fibronectin, and MMP9 expression levels, when compared with their respective controls (Fig. 5A and B).

To determine if GCIP suppression of NSCLC progression may be mediated by inhibiting the Id1 signaling pathway, a stable Tet-On system for doxycycline-dependent expression of GCIP was established in A549/Id1 cells (Supplementary Fig. S6C). Expression of p-PI3K (Tyr467), p-Akt (Ser473), NFкB/ p65, snail, slug, vimentin, and MMP9, which was elevated by overexpressing Id1 in A549/Id1/Tet-on-GCIP cells (clone -1 and -2), was profoundly decreased when GCIP was expressed (Fig. 5C and D). Taken together, these data reveal that GCIP suppresses NSCLC progression is, at least in part, mediated by inhibiting Id1 downstream signaling.

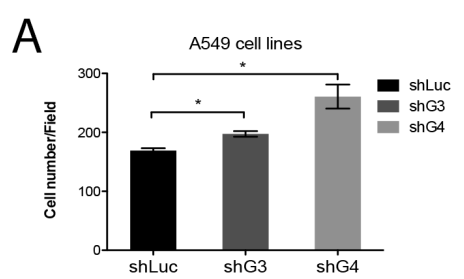

B

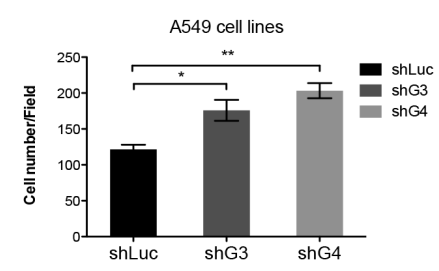

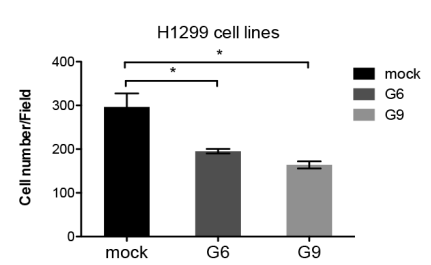

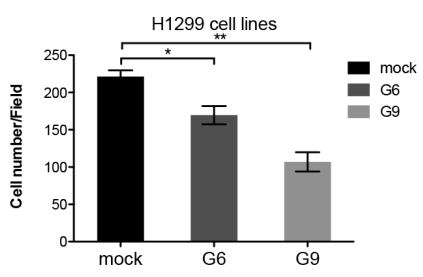

C
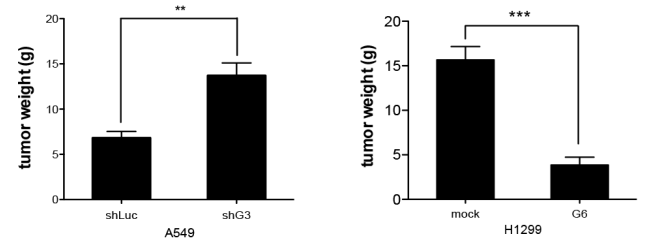

D

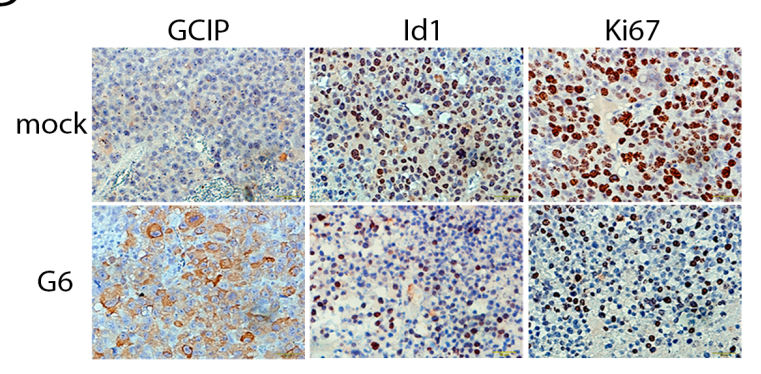

Figure 4: GCIP decreases and Id1 increases the migration and invasion of NSCLC cancer cells in vitro and GCIP suppresses tumor growth in vivo. Effects of GCIP on the migration and invasion of NSCLC cell lines. A, A549 (left) or H1299 (right) stable clones were placed in the upper chamber of a Trans-well and allowed to migrate for $24 \mathrm{~h}$. B, A549 (left) or H1299 (right) stable clones were placed in the upper Matrigel invasion chamber and allowed to migrate for $24 \mathrm{~h}$. Cells that had invaded to the bottom of the membrane were counted manually after staining with crystal violet. C, GCIP-mediated control of in vivo tumorigenesis. For each injection, stable A549/shLuc, A549/shGCIP-3, H1299/mock, H1299/GCIP-6 cells were subcutaneously implanted into 4 to 6-week-old female NOD/SCID mice. Five mice were used for each group (points, mean; bars, SEM). D, sections of tumors from mice were analyzed for GCIP, Id1, and Ki67 expression by immunohistochemistry ( $\times 40$ magnification). ${ }^{* * *, * * *} P \leq 0.05$ based on Student's t-test analysis. 


\section{GCIP and Id1 are inversely expressed in NSCLC cell lines and tissues}

Although Id1 overexpression and silencing did not alter GCIP expression in A549/Id1 and H1299/shId1 cells, respectively (Supplementary Fig. S7A), we observed a marked decrease in Id1 expression in the GCIPoverexpressing (H1299/GCIP-9) cells and a significant increase in Id1 expression in the GCIP-silenced (A549/ shGCIP-3 and -4) cells (Supplementary Fig. S7B). Cotransfection of an $I d 1$ promoter reporter construct (from -1 to -2134) [13] with a GCIP expression plasmid, a GCIP-shRNA plasmid, or control vector, revealed that ectopic expression of GCIP led to markedly decreased Id 1 promoter reporter activity; expression of shGCIP led to upregulated $I d I$ promoter reporter activity (Fig. 6A). Thus, GCIP expression downregulates Id1 expression by inhibiting its transcription, and silencing GCIP upregulates Id1 expression in NSCLC cell lines.

The inverse correlation between GCIP and Id1 expression was also observed in NSCLC specimens. As shown in Fig. 6B, Id1 was weakly expressed in early-stage NSCLC specimens; however, its expression increased with malignancy. Furthermore, Id1 expression analysis was also conducted using the same samples employed for GCIP IHC analysis in Fig. 1B. Low levels of GCIP expression significantly correlated with high levels of Id1 expression in the metastatic tumors (Fig. 6C). This inverse expression pattern between GCIP and Id1 was observed in the NSCLC tissues from 72 human patients; there was a statistically significant inverse correlation between GCIP and Id1 expression $(\mathrm{r}=-0.2834, p<0.05)$. A previous study reported that higher Id1 levels were associated with an overall survival in adenocarcinoma but not in squamous cell carcinoma from 346 NSCLC patients[15], however survival analysis using the Kaplan-Meier plotter database (www.kmplot.com, 2014 version) with a log-rank test revealed that increased Id1 expression correlated with poor prognosis in both the adenocarcinoma and squamous cell carcinoma from 1405 NSCLC patients (Fig. 6D and Supplementary Fig. S7C). Nevertheless no information of GCIP expression can be seen in this database. Taken together, GCIP inhibits the expression of Id1, and GCIP and Id1 are inversely expressed in NSCLC cell lines and tissue specimens, suggesting that GCIP suppresses NSCLC progression by downregulating the expression and function of Id1.
A

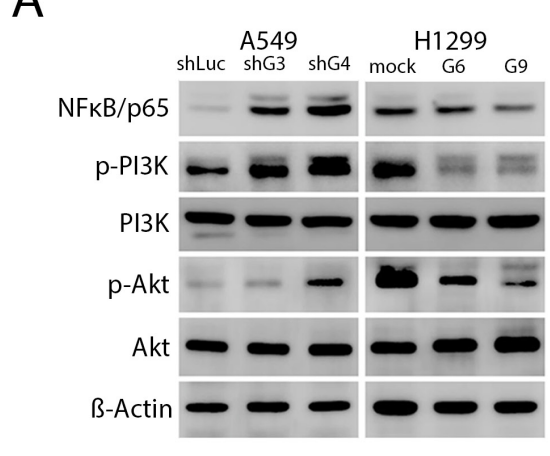

C

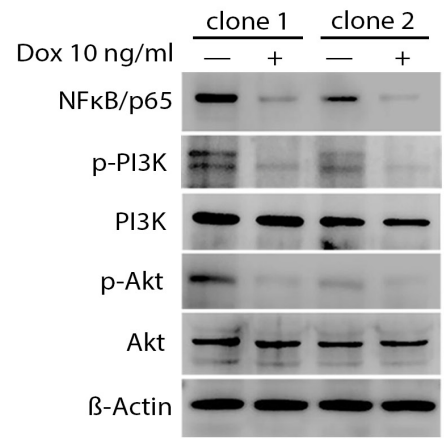

B

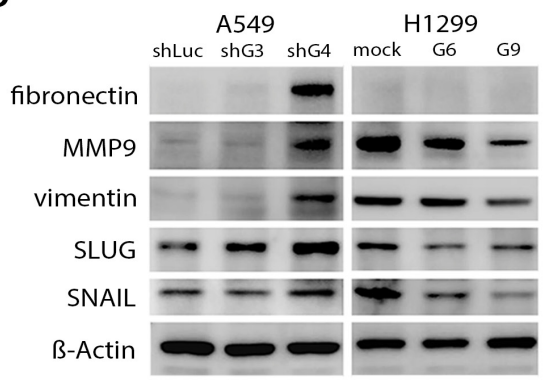

D

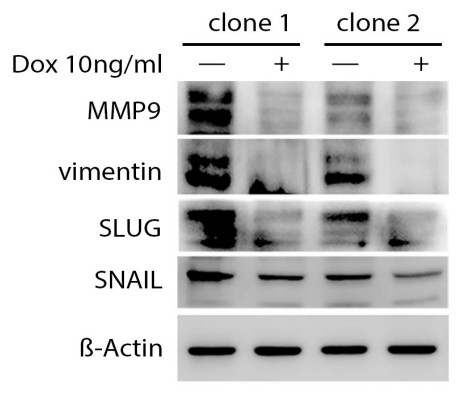

Figure 5: Inhibition of Id-1-induced PI3K/Akt/NFאB signaling- and EMT-associated proteins by GCIP. A, The expression of PI3K, phosphorylated PI3K (p-PI3K), Akt, phosphorylated Akt (p-Akt) and NFkB/p65 in A549 and H1299 stable clones. The A549 stable clones had markedly higher expression of p-PI3K, p-Akt and NFKB/p65 than the H1299 stable clones. B, immunoblot analysis showed reduced expression of EMT markers in H1299 stable cells compared with vector control cells and the A549 stable clones. C, Expression of PI3K/Akt/NFKB signaling proteins in doxycycline-induced cells in the presence of Id1. D, MMP9, vimentin, SLUG and SNAIL protein expression was detected by immunoblot assays using specific antibodies. GCIP expression was induced by growing cells in the presence of $10 \mathrm{ng} / \mathrm{mL}$ doxycycline for 2 days. Samples were normalized for levels of $\beta$-actin. 


\section{Effects of GCIP on sensitivity of NSCLC cells to chemotherapy}

Because increased Id 1 expression was correlated with chemoresistance of NSCLC cells [15], we examined whether overexpression of GCIP could reverse this chemoresistance. GCIP-overexpressing H1299 cells (H1299/GCIP-9), GCIP-silenced A549 cells (A549/ shGCIP-4), or their respective control cells were treated with 0,25 and $200 \mu \mathrm{g} / \mathrm{mL}$ carboplatin for $72 \mathrm{~h}$. As expected, H1299/GCIP-9 cells treated with $200 \mu \mathrm{g} /$ $\mathrm{mL}$ carboplatin exhibited a significant reduction in cell growth compared to the H1299 cells treated with the same dose $(p<0.05$; Figure 6F). On the contrary, A549/ shGCIP-4 cells exhibited a significantly increased the cell viability when treated with 25 or $200 \mu \mathrm{g} / \mathrm{ml}$ carboplatin compared to the A549 cells treated with the same dose $(p<0.05$; Fig. 6E). Similar results were also obtained from these cells treated with docetaxel, which has been approved as a first- and second-line treatment for advanced NSCLC (Supplementary Fig. S7D and E). Thus, depletion of GCIP promoted resistance, while expression of GCIP potentiated the cytotoxic effect of anticancer drugs, suggesting that GCIP expression increased susceptibility to chemotherapeutic agents by inhibiting Id1-induced chemoresistance. Furthermore, depletion of GCIP, which was observed in most NSCLC specimens especially in patients with invasive NSCLC, might reduce chemosensitivity in NSCLC patients.

\section{DISCUSSION}

Growing evidence indicates that the GCIP is a novel tumor suppressor in breast, colon and liver cancers [22,
A

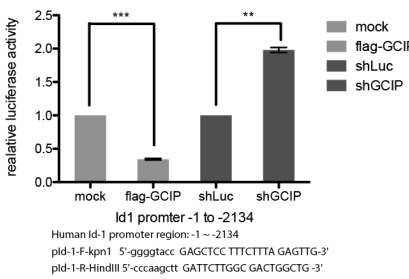

B

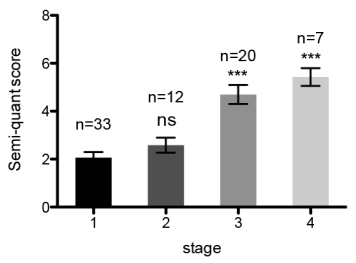

NSCLC: Adenocarcinoma

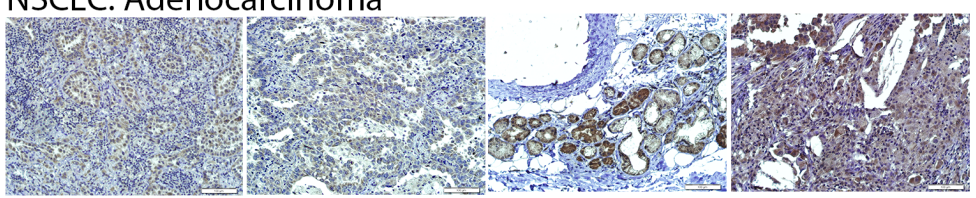

NSCLC: Squamouse cell carcinoma

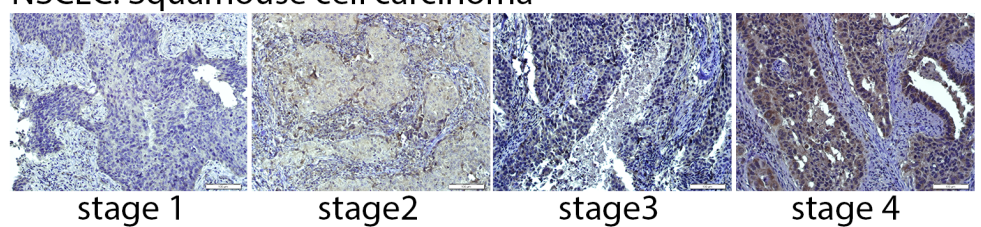

E

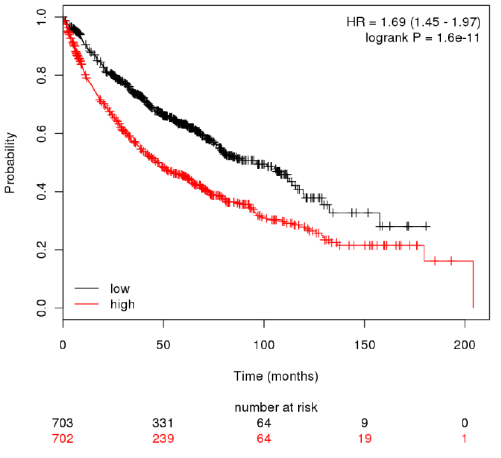

$\mathrm{F}$
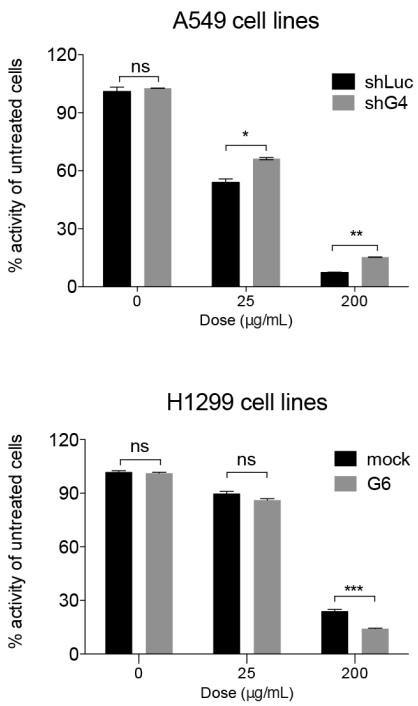

Figure 6: GCIP downregulation of Id1 mRNA expression in NSCLC cells. A, A549 cells were triple-transfected with the full-length $I d 1$ promoter reporter construct, pSV- $\beta$-Galactosidase plus Flag-GCIP or shGCIP. Cells were then incubated in 10\% FBS DMEM for $24 \mathrm{~h}$. The primers used for the construction of the $I d l$ promoter are indicated at the bottom. B, quantification of Id1 expression by IHC analysis of lung cancer specimens. The number (n) of samples for each stage is indicated on the top. C, Id1 expression levels in adenocarcinoma (upper panel) and squamous cell carcinoma (lower panel) of NSCLC specimens by immunohistochemical analysis (magnification x20). Scale bars, $100 \mu \mathrm{m}$. Higher Id1 expression levels in NSCLC stages III and IV were observed compared with stage I tissues. Scale bars, $100 \mu \mathrm{m}$. D, the data from Kaplan-Meier overall survival analysis shows that Id1 is associated with poor prognosis. ${ }^{* * *} P<0.001$ based on Student's t-test. GCIP increased sensitivity to carboplatin in NSCLC cell lines. E, A549/shLuc, A549/shGCIP-4 cells were treated with 0,25 and $200 \mu \mathrm{g} / \mathrm{mL}$ carboplatin. F, H1299/mock, H1299/GCIP-9 cells were treated with 0, 25 and $200 \mu \mathrm{g} / \mathrm{mL}$ carboplatin. Cell proliferation was measured using an MTT assay ( $72 \mathrm{~h}$ after treatment) and showed that GCIP upregulation significantly sensitized cells to therapy. ${ }^{*} P<0.05 ;{ }^{* *} P<0.01 ;{ }^{* * *} P<0.001$; ns: not significant. 
27-29]. However, the precise mechanisms underlying its anti-tumor properties have not been fully elucidated. It is generally thought that GCIP may inhibit the protein expression and function of cyclin D1, and thereby, inhibiting cell proliferation and tumorigenesis $[18,20,22-$ $24,27,28]$. Consistent with these previous studies, in this study, we also found that both cyclin D1 expression and phosphorylation of $\mathrm{Rb}$ at Ser780 (RbS780) were enhanced in GCIP-silenced A549 cells; both cyclin D1 and RbS780 were decreased in GCIP-overexpressing H1299 cells (data not shown). Furthermore, our data presented here also demonstrated that GCIP interacts with and represses Id in NSCLC cell lines. Since cyclin D1 has been implication in several cell types as a target of Id1 $[33,34]$ and is a key modulator proliferation, it is reasonable to speculate that attenuation of GCIP would lead to upregulation of Id1, which may also contribute to enhanced cyclin D1 and RbS780 levels, subsequently leading to the increased proliferation.

In addition to inducing cell proliferation, Id1 may also induce EMT, inhibit apoptosis and promote migration, invasion, metastasis and drug resistance in several types of cancer [35, 36]. It is an upstream regulator of the $\mathrm{PI} 3 \mathrm{~K} / \mathrm{Akt} / \mathrm{NF}$ KB pathway in esophageal, head and neck, ovarian, gastric and lung cancer cells [32, 37, 38], and the expression of vimentin and fibronectin is regulated by Id1 at transcriptional level in NSCLC [16]. In addition, Id1 induces snaillexpression in kidney epithelial cells [39] and positively regulates MMP-9 expression in breast and lung cancer cell lines [40, 41]. Consistent with these previous studies, Id1-induced expression and/or activation of the aforementioned proteins was inhibited in GCIPoverexpressing H1299 cells, and was enhanced in GCIPsilenced A549 cells. Furthermore, GCIP downregulated these proteins through inhibition of Id1, which was confirmed in a conditionally induced stable A549 cell line. Significantly higher Id 1 protein expression was previously found in 346, 61[15, 41] and 1405 NSCLC specimens (www.kmplot.com, 2014 version), respectively, and 72 NSCLC specimens in this study. As compared to Id3, more frequent expression of Id1 was observed in NSCLC specimens [42]. Furthermore, among the Id proteins, GCIP exhibited the strongest interaction with Id1, inhibiting its expression. Given the role of the PI3K/Akt/NFkB pathway in apoptosis resistance [38, 43], chemoresistance [44, 45] as well as the regulation of cellular invasion and metastasis $[46,47]$ as well as the importance of both snail and Slug as critical regulators of EMT, which is important for tumor invasion and metastasis, and resistance to several antitumor-drugs [48, 49] combined with the role of MMP9 as an important mediator of malignant invasiveness and metastasis [40], it is tempting to speculate that GCIPmediated downregulation of Id1 and its downstream effectors could be a key mechanism by which GCIP can potently suppress NSCLC tumor progression.

Id1 is recognized as an inhibitor of differentiation and essential for embryonic development [6]. Consistent with dedifferentiation of adult tissues being intricately connected to oncogenesis, the evidence for an important role of Id1 in cancer development and progression seems to be growing [35]. Alternatively, although GCIP is also recognized as a dnHLH protein and structurally related to the mouse Id1-like molecule, Maid, it is widely expressed in differentiated cells in adults and has been demonstrated to be a tumor suppressor in breast, colon and liver cancers in contrast to the extremely low expression of Id1 in adult tissues. Induction of Id1 expression in response to extracellular signaling molecules, including bone morphogenetic proteins (BMPs), has also been reported $[31,50,51]$. In addition, previous studies have also demonstrated that Id1 is required to maintain self-renewal capacity in various stem cells $[13,52]$, and is required for tumor initiating function, both in the context of primary tumor formation and during metastatic colonization of the lung microenvironment [53]. In this study, we demonstrated that GCIP transcriptionally inhibited IdI expression and exerted antagonistic effects on Id1-driven tumor progression of NSCLC. On the basis of these findings, and the fact that GCIP is expressed mainly in terminally differentiated tissues in adults where Id1 levels are extremely low, we propose that GCIP expression in both the non-transformed lung cells and the differentiated NSCLC cells serves to restrict the levels of Id1. However, in the presence of extracellular signaling molecules that can induce Id1 but not GCIP expression in NSCLC cell lines (e.g., BMP2, Fig. 1C), upregulation of Id1 may overcome the tumor suppressive functions of GCIP. In addition, loss of GCIP through currently unidentified mechanisms during tumor development could also contribute to enhanced Id 1 levels, consequently promoting tumor progression of NSCLC. The regulation of GCIP and the mechanisms that GCIP transcriptionally regulates Id1 are currently being studied.

Our present study also showed that GCIP expression level negatively correlated with NSCLC disease stage, and that GCIP and Id 1 are inversely expressed in NSCLC cell lines and tissues. In addition, we also demonstrated that GCIP downregulated Id1 to render NSCLC cells more susceptibility to carboplatin and docetaxel, two commonly used anticancer drugs for NSCLC. Thus, GCIP may also be a useful prognostic marker for NSCLC in addition to Id1. Furthermore, our data also imply that robust induction of GCIP may not only improve chemosensitivity but also be an effective therapeutic approach in NSCLC.

In summary, GCIP is a tumor suppressor that interacts with Id1 in NSCLC. Upregulation of GCIP suppresses Id1-mediated downstream signaling. Clinical and mechanistic evidence supports that GCIP exerts its tumor-suppressive function in NSCLC through suppression of Id1-triggered oncogenic properties. 


\section{MATERIALS AND METHODS}

\section{Cell lines}

$293 \mathrm{~T}$ cells and the lung cancer cell lines, A549, H1299, CL1-0 and CL1-5, were cultured in complete medium consisting of Dulbecco's Modified Eagle's medium (DMEM, Gibco, Grand Island, NY, USA) and $10 \%$ fetal bovine serum (FBS, Invitrogen, Carlsbad, CA, USA). All cells were incubated in $10-\mathrm{cm}$ tissue culture dishs (BD Falcon, San Jose, CA, USA) at $37^{\circ} \mathrm{C}$ and $5 \%$ $\mathrm{CO}_{2}$, and were subcultured every 3-4 days. P.C. Yang kindly provided the lung cancer cell lines, CL1-0 and CL1-5. The lung cancer cell lines, A549 and H1299, were purchased from Bioresource Collection and Research Center (BCRC), Hsinchu, Taiwan.

\section{Immunoprecipitation}

Cells that were transfected with plasmids as described in Supplementary Methods were washed with cold PBS and lysed in NP-40 buffer ( $\mathrm{NaCl}, 150 \mathrm{mM}$, NP$40,1.0 \%$, Tris- $\mathrm{Cl} 50 \mathrm{mM}, \mathrm{pH}$ 8.0). A detailed procedure for the immunoprecipitation assay was described in the Supplementary Data.

\section{GST pull-down assay}

BL-21 bacteria were transformed with pGEX-5X-3 (GST alone) or each GST fusion construct a described in Supplementary Methods. A detailed procedure for the GST pull-down assay was described in the Supplementary Data.

\section{Id1 promoter assays}

Luciferase and galactosidase assay systems (Promega, Madison, WI, USA) were used for promoter analysis. A detailed procedure for the Id1 promoter analysis with GCIP was described in the Supplementary Data.

\section{Patient clinical specimens and immunohistochemistry}

NSCLC tissue samples were collected from 72 consecutive patients, who underwent resection at the National Cheng Kung University Hospital. All patients gave informed consent for retention and analysis of their tissue for research. Unstained slides were deparaffinized in xylene and rehydrated sequentially in ethanol. Immunohistochemistry analysis of GCIP was performed as previously described[29] and Id1 was described in the
Supplementary Data.

\section{Proliferation, migration, invasion and clonogenic assays}

Cell proliferation was measured by trypan-blue staining. Briefly, cells were plated at a concentration of $1 \times 10^{5}$ cells in $10-\mathrm{cm}$ dishes. Every $24 \mathrm{~h}$, cells were trypsinized and counted. To evaluate migration or invasion, $1-5 \times 10^{4}$ cells were cultured in serum-free DMEM in an upper compartment of a transwell (BD Biosciences) or with a matrigel-coated transwell (BD Biosciences) using 24-well plates for 24 to $72 \mathrm{~h}$. The wiped membrane was fixed and stained with $0.5 \%$ crystal violet. The percentages of migrating cells were calculated in three independent representative fields. Colony formation assays were conducted by plating 500 cells/well into $100-\mathrm{mm}$ culture dishes in triplicate. After a 12 -day incubation at $37^{\circ} \mathrm{C}$ and $5 \% \mathrm{CO}_{2}$, cells were fixed with formaldehyde and stained with $2 \%$ crystal violet. The number of colonies was then counted, and the surviving fraction of treated cells was normalized to the surviving fraction of the corresponding controls.

\section{Xenograft}

To examine the effect of GCIP expression in tumor formation, $1 \times 10^{6} \mathrm{H} 1299 /$ mock, H1299/G6, A549/shLuc or A549/shG3 cells were implanted subcutaneously into 5-week-old female BALB/c nude mice $(n=5)$, and the tumor growth was monitored using calipers every 3 to 4 days. Animals were sacrificed 30 or 35 days after inoculation, and tumors were isolated and fixed in $10 \%$ natural-buffer formalin (Merck, Whitehouse Station, NJ, USA) for further immunohistochemical staining was performed as previously described [29].

\section{Expression dataset from microarray analysis}

The Oncomine databases were queried to identify the prognostic role of GCIP components in NSCLC. The Oncomine Cancer Microarray database from Hou et al. [30] was employed to compare GCIP gene expression in normal lung and primary in NSCLC tumor.

\section{Cell viability analysis by MTT assay}

A549/shLuc, A549/shG3, H1299/mock and H1299/ G6 cells were plated in 96-well plates $\left(2 \times 10^{6}\right.$ cells $\left./ \mathrm{mL}\right)$ in triplicate. Cells were treated with 0,25 , and $200 \mu \mathrm{g} /$ $\mathrm{mL}$ carboplatin or $0,25 \mu \mathrm{g} / \mathrm{mL}$ docetaxel. After 3 days, cell proliferation was measured using MTT (Sigma, St. Louis, MO, USA) assays according to the manufacturer's protocol. Absorbance was measured at $490 \mathrm{~nm}$. 


\section{Scoring and statistical analysis}

Staining intensities $(1=+, 2=++$ and $3=+++)$, fraction of positive cells $(0=0 \%, 1=<5 \%, 2=5-50 \%$ and $3=>50 \%$ of tumor cells) and the histological grade (G1 = well, $\mathrm{G} 2=$ moderate and G3 = poor) were scored by an experienced pathologist (MG), who was blinded to the patient's clinical details. The semi-quantitative $\mathrm{H}$-score, defined as the staining intensity $\times$ fraction of positive cells (range, $0-9$ ), was determined and used for statistical analysis. Pearson rank correlation (r) was used to assess correlation between $G C I P$ and $I d I$ genes. Unless otherwise specified, all experiments were performed in triplicate and were repeated at least twice. Data were expressed as mean values $\pm \mathrm{SE}$ or $\mathrm{SD}$ and were analyzed using GraphPad Prism software. The level of significance was set at $P<0.05$.

\section{ACKNOWLEDGEMENTS}

The authors thank Yi-Ying Chien, Yu-Jhen Yan, Han-Chun Pan, Jennifer Cheng and Dr. Po-Lin Chen for technical assistance in National Cheng Kung University as well as the National RNAi Core Facility located at the Institute of Molecular Biology/Genomic Research Center, Academia Sinica for assistance in lentivirus production. We are grateful to Sheng-Hsiang Lin and Jia-Ling Wu for providing the statistical consulting services from the Biostatistics Consulting Center, National Cheng Kung University Hospital.

\section{Grant support}

This work was supported by a grant from the National Science Council, Taiwan (97-2311-B-006-005MY3).

\section{REFERENCES}

1. Katakami N, Sugiura T, Nogami T, Yamamoto H, Negoro S, Nakano T, Okamoto N, Takada Y, Kodama K and Ariyoshi Y. Combination chemotherapy of gemcitabine and vinorelbine for patients in stage IIIB-IV non-small cell lung cancer: a phase II study of the West Japan Thoracic Oncology Group (WJTOG) 9908. Lung cancer. 2004; 43(1):93-100.

2. Dasgupta P, Kinkade R, Joshi B, Decook C, Haura E and Chellappan S. Nicotine inhibits apoptosis induced by chemotherapeutic drugs by up-regulating XIAP and survivin. Proceedings of the National Academy of Sciences of the United States of America. 2006; 103(16):6332-6337.

3. Siegel R, Naishadham D and Jemal A. Cancer statistics, 2013. CA: a cancer journal for clinicians. 2013; 63(1):1130.
4. Signe Søes ILD, Brita Singers Sørensen, Andreas Carus, Manuel Mattheisen, Jan Alsner, Jens Overgaard, Henrik Hager, Lise Lotte Hansen, Lasse Sommer Kristensen. Hypomethylation and increased expression of the putative oncogene ELMO3 are associated with lung cancer development and metastases formation. Oncoscience. 2014; $1(5)$. in press.

5. Kyoko Okamura KT, Kohichi Kawahara, Taishi Harada, Miki Nishio, Kohei Otsubo, Kayo Ijichi, Mikihiro Kohno, Eiji Iwama, Akiko Fujii, Keiichi Ota, Takaomi Koga, Tatsuro Okamoto, Akira Suzuki, Yoichi Nakanishi. PICT1 expression is a poor prognostic factor in non-small cell lung cancer. Oncoscience. 2014; 2014; 1(5): in press.

6. Ruzinova MB and Benezra R. Id proteins in development, cell cycle and cancer. Trends in Cell Biology. 2003; 13(8):410-418.

7. Y.-C. Wong XWaM-TL. Id-1 expression and cell survival. Apoptosis 2004; 9:279-289.

8. Jean-Philippe Coppe YI, Dan H. Moore JLB, and and Desprez P-Y. Id-1 and Id-2 Proteins as Molecular Markers for Human Prostate Cancer Progression. Clinical Cancer Research. 2004; 10:2044-2051.

9. Fong S, Itahana Y, Sumida T, Singh J, Coppe JP, Liu Y, Richards PC, Bennington JL, Lee NM, Debs RJ and Desprez PY. Id-1 as a molecular target in therapy for breast cancer cell invasion and metastasis. Proceedings of the National Academy of Sciences of the United States of America. 2003; 100(23):13543-13548.

10. Anido J, Saez-Borderias A, Gonzalez-Junca A, Rodon L, Folch G, Carmona MA, Prieto-Sanchez RM, Barba I, Martinez-Saez E, Prudkin L, Cuartas I, Raventos C, Martinez-Ricarte F, Poca MA, Garcia-Dorado D, Lahn MM, et al. TGF-beta Receptor Inhibitors Target the CD44(high)/Id1(high) Glioma-Initiating Cell Population in Human Glioblastoma. Cancer Cell. 2010; 18(6):655-668.

11. Perk J, Gil-Bazo I, Chin Y, de Candia P, Chen JJ, Zhao Y, Chao S, Cheong W, Ke Y, Al-Ahmadie H, Gerald WL, Brogi E and Benezra R. Reassessment of id1 protein expression in human mammary, prostate, and bladder cancers using a monospecific rabbit monoclonal anti-id1 antibody. Cancer research. 2006; 66(22):10870-10877.

12. Monika Schindl GO, Andreas Obermair, Sebastian F. Schoppmann, Barbara Karner, and Peter Birner. Overexpression of Id-1 Protein Is a Marker for Unfavorable Prognosis in Early-Stage Cervical Cancer. Cancer research. 2001; 61:5703-5706.

13. Soroceanu L, Murase R, Limbad C, Singer E, Allison J, Adrados I, Kawamura R, Pakdel A, Fukuyo Y, Nguyen D, Khan S, Arauz R, Yount GL, Moore DH, Desprez PY and McAllister SD. Id-1 is a key transcriptional regulator of glioblastoma aggressiveness and a novel therapeutic target. Cancer research. 2013; 73(5):1559-1569.

14. Minn AJ, Gupta GP, Siegel PM, Bos PD, Shu W, Giri DD, Viale A, Olshen AB, Gerald WL and Massague J. Genes that mediate breast cancer metastasis to lung. Nature. 2005; 
436(7050):518-524.

15. Ponz-Sarvise M, Nguewa PA, Pajares MJ, Agorreta J, Lozano MD, Redrado M, Pio R, Behrens C, Wistuba, II, Garcia-Franco CE, Garcia-Foncillas J, Montuenga LM, Calvo A and Gil-Bazo I. Inhibitor of differentiation-1 as a novel prognostic factor in NSCLC patients with adenocarcinoma histology and its potential contribution to therapy resistance. Clinical cancer research : an official journal of the American Association for Cancer Research. 2011; 17(12):4155-4166.

16. Pillai S, Rizwani W, Li X, Rawal B, Nair S, Schell MJ, Bepler G, Haura E, Coppola D and Chellappan S. ID1 facilitates the growth and metastasis of non-small cell lung cancer in response to nicotinic acetylcholine receptor and epidermal growth factor receptor signaling. Molecular and cellular biology. 2011; 31(14):3052-3067.

17. Ishii R, Isogaya $\mathrm{K}$, Seto A, Koinuma D, Watanabe $\mathrm{Y}$, Arisaka F, Yaguchi S, Ikushima H, Dohmae N, Miyazono K, Miyazawa K, Ishitani R and Nureki O. Structure of a dominant-negative helix-loop-helix transcriptional regulator suggests mechanisms of autoinhibition. EMBO J. 2012; 31(11):2541-2552.

18. Xia C, Bao Z, Tabassam F, Ma W, Qiu M, Hua S and Liu M. GCIP, a novel human grap2 and cyclin D interacting protein, regulates E2F-mediated transcriptional activity. The Journal of biological chemistry. 2000; 275(27):2094220948.

19. Shuji Terai HA, Kozo Ashida and Snorri S. Human homologue of maid: A dominant inhibitory helix-loophelix protein associated with liver-specific gene expression. Hepatology. 2000; 32(2):357-366.

20. Yao Y, Doki Y, Jiang W, Imoto M, Venkatraj VS, Warburton D, Santella RM, Lu B, Yan L, Sun XH, Su $\mathrm{T}$, Luo $\mathrm{J}$ and Weinstein IB. Cloning and characterization of DIP1, a novel protein that is related to the Id family of proteins. Experimental cell research. 2000; 257(1):22-32.

21. Chang MS, Chang CL, Huang CJ and Yang YC. p29, a novel GCIP-interacting protein, localizes in the nucleus. Biochemical and biophysical research communications. 2000; 279(2):732-737.

22. Ma W, Stafford LJ, Li D, Luo J, Li X, Ning G and Liu M. GCIP/CCNDBP1, a helix-loop-helix protein, suppresses tumorigenesis. Journal of cellular biochemistry. 2007; 100(6):1376-1386.

23. Lee I, Yeom SY, Lee SJ, Kang WK and Park C. A novel senescence-evasion mechanism involving Grap2 and Cyclin D interacting protein inactivation by Ras associated with diabetes in cancer cells under doxorubicin treatment. Cancer research. 2010; 70(11):4357-4365.

24. Chang TW, Chen CC, Chen KY, Su JH, Chang JH and Chang MC. Ribosomal phosphoprotein P0 interacts with GCIP and overexpression of P0 is associated with cellular proliferation in breast and liver carcinoma cells. Oncogene. 2008; 27(3):332-338.
25. Ikushima H KA, Isogaya K, Shinozaki M, Hellman U, Miyazawa K, Miyazono K. An Id-like molecule, HHM, is a synexpression group-restricted regulator of TGF-b signalling. The EMBO Journal. 2008; 27:2955-2965.

26. Xiang H, Wang J, Mao Y, Liu M, Reddy VN and Li DW. Human telomerase accelerates growth of lens epithelial cells through regulation of the genes mediating $\mathrm{RB} / \mathrm{E} 2 \mathrm{~F}$ pathway. Oncogene. 2002; 21(23):3784-3791.

27. Ma W, Xia X, Stafford LJ, Yu C, Wang F, LeSage G and Liu M. Expression of GCIP in transgenic mice decreases susceptibility to chemical hepatocarcinogenesis. Oncogene. 2006; 25(30):4207-4216.

28. Sonnenberg-Riethmacher E, Wustefeld T, Miehe M, Trautwein C and Riethmacher D. Maid (GCIP) is involved in cell cycle control of hepatocytes. Hepatology. 2007; 45(2):404-411.

29. Chen WC, Su PF, Jin YT, Chang MC and Chang TW. Immunohistochemical expression of GCIP in breast carcinoma: relationship with tumour grade, diseasefree survival, mucinous differentiation and response to chemotherapy. Histopathology. 2008; 53(5):554-560.

30. Hou J, Aerts J, den Hamer B, van Ijcken W, den Bakker M, Riegman P, van der Leest C, van der Spek P, Foekens JA, Hoogsteden HC, Grosveld F and Philipsen S. Gene expression-based classification of non-small cell lung carcinomas and survival prediction. PloS one. 2010; 5(4):e10312.

31. Korchynskyi $\mathrm{O}$ and ten Dijke P. Identification and functional characterization of distinct critically important bone morphogenetic protein-specific response elements in the Id1 promoter. The Journal of biological chemistry. 2002; 277(7):4883-4891.

32. Cheng YJ, Tsai JW, Hsieh KC, Yang YC, Chen YJ, Huang MS and Yuan SS. Id1 promotes lung cancer cell proliferation and tumor growth through Akt-related pathway. Cancer letters. 2011; 307(2):191-199.

33. Swarbrick A, Akerfeldt MC, Lee CS, Sergio CM, Caldon CE, Hunter LJ, Sutherland RL and Musgrove EA. Regulation of cyclin expression and cell cycle progression in breast epithelial cells by the helix-loop-helix protein Id1. Oncogene. 2005; 24(3):381-389.

34. Caldon CE, Swarbrick A, Lee CS, Sutherland RL and Musgrove EA. The helix-loop-helix protein Id1 requires cyclin D1 to promote the proliferation of mammary epithelial cell acini. Cancer research. 2008; 68(8):30263036.

35. Ling MT, Wang X, Zhang X and Wong YC. The multiple roles of Id-1 in cancer progression. Differentiation; research in biological diversity. 2006; 74(9-10):481-487.

36. Zhang X, Ling MT, Wang Q, Lau CK, Leung SC, Lee TK, Cheung AL, Wong YC and Wang X. Identification of a novel inhibitor of differentiation-1 (ID-1) binding partner, caveolin-1, and its role in epithelial-mesenchymal transition and resistance to apoptosis in prostate cancer cells. The 
Journal of biological chemistry. 2007; 282(46):3328433294.

37. Li B, Cheung PY, Wang X, Tsao SW, Ling MT, Wong YC and Cheung AL. Id-1 activation of PI3K/Akt/NFkappaB signaling pathway and its significance in promoting survival of esophageal cancer cells. Carcinogenesis. 2007; 28(11):2313-2320.

38. Lin J, Guan Z, Wang C, Feng L, Zheng Y, Caicedo E, Bearth E, Peng JR, Gaffney P and Ondrey FG. Inhibitor of differentiation 1 contributes to head and neck squamous cell carcinoma survival via the NF-kappaB/survivin and phosphoinositide 3-kinase/Akt signaling pathways. Clinical cancer research : an official journal of the American Association for Cancer Research. 2010; 16(1):77-87.

39. Li Y, Wen X and Liu Y. Tubular cell dedifferentiation and peritubular inflammation are coupled by the transcription regulator Id1 in renal fibrogenesis. Kidney international. 2012; 81(9):880-891.

40. Sun X, Li C, Zhuang C, Gilmore WC, Cobos E, Tao $\mathrm{Y}$ and Dai Z. Abl interactor 1 regulates Src-Id1-matrix metalloproteinase 9 axis and is required for invadopodia formation, extracellular matrix degradation and tumor growth of human breast cancer cells. Carcinogenesis. 2009; 30(12):2109-2116.

41. Rothschild SI, Kappeler A, Ratschiller D, Betticher DC, Tschan MP, Gugger M and Gautschi O. The stem cell gene "inhibitor of differentiation 1" (ID1) is frequently expressed in non-small cell lung cancer. Lung cancer. 2011; 71(3):306-311.

42. Castanon E, Bosch-Barrera J, Lopez I, Collado V, Moreno M, Lopez-Picazo JM, Arbea L, Lozano MD, Calvo A and Gil-Bazo I. Id 1 and Id 3 co-expression correlates with clinical outcome in stage III-N2 non-small cell lung cancer patients treated with definitive chemoradiotherapy. Journal of translational medicine. 2013; 11:13.

43. Vasudevan KM, Gurumurthy $\mathrm{S}$ and Rangnekar VM. Suppression of PTEN Expression by NF- B Prevents Apoptosis. Molecular and cellular biology. 2004; 24(3):1007-1021.

44. LoPiccolo J, Blumenthal GM, Bernstein WB and Dennis PA. Targeting the PI3K/Akt/mTOR pathway: effective combinations and clinical considerations. Drug resistance updates : reviews and commentaries in antimicrobial and anticancer chemotherapy. 2008; 11(1-2):32-50.

45. Trevino JG, Pillai S, Kunigal S, Singh S, Fulp WJ, Centeno BA and Chellappan SP. Nicotine induces inhibitor of differentiation-1 in a Src-dependent pathway promoting metastasis and chemoresistance in pancreatic adenocarcinoma. Neoplasia (New York, NY). 2012; 14(12):1102-1114.

46. Bader AG, Kang S, Zhao L and Vogt PK. Oncogenic PI3K deregulates transcription and translation. Nature reviews Cancer. 2005; 5(12):921-929.

47. Karin M, Cao Y, Greten FR and Li ZW. NF-kappaB in cancer: from innocent bystander to major culprit. Nature reviews Cancer. 2002; 2(4):301-310.

48. Bolos V, Peinado H, Perez-Moreno MA, Fraga MF, Esteller $M$ and Cano A. The transcription factor Slug represses E-cadherin expression and induces epithelial to mesenchymal transitions: a comparison with Snail and E47 repressors. J Cell Sci. 2003; 116(Pt 3):499-511.

49. Shih JY and Yang PC. The EMT regulator slug and lung carcinogenesis. Carcinogenesis. 2011; 32(9):1299-1304.

50. Belletti B, Prisco M, Morrione A, Valentinis B, Navarro $\mathrm{M}$ and Baserga R. Regulation of Id 2 gene expression by the insulin-like growth factor I receptor requires signaling by phosphatidylinositol 3-kinase. The Journal of biological chemistry. 2001; 276(17):13867-13874.

51. Ying QL, Nichols J, Chambers I and Smith A. BMP induction of Id proteins suppresses differentiation and sustains embryonic stem cell self-renewal in collaboration with STAT3. Cell. 2003; 115(3):281-292.

52. Romero-Lanman EE, Pavlovic S, Amlani B, Chin Y and Benezra R. Id1 maintains embryonic stem cell self-renewal by up-regulation of Nanog and repression of Brachyury expression. Stem cells and development. 2012; 21(3):384393.

53. Gupta GP, Perk J, Acharyya S, de Candia P, Mittal V, Todorova-Manova K, Gerald WL, Brogi E, Benezra R and Massague J. ID genes mediate tumor reinitiation during breast cancer lung metastasis. Proceedings of the National Academy of Sciences of the United States of America. 2007; 104(49):19506-19511. 